\title{
La recherche en éducation
}

Entretien avec Antoine Prost

Antoine Prost et Odile Luginbühl

\section{CpenEdition}

\section{Journals}

Édition électronique

URL : https://journals.openedition.org/ries/1939

DOI : 10.4000/ries. 1939

ISSN : 2261-4265

Éditeur

France Education international

Édition imprimée

Date de publication : 1 avril 2002

Pagination : 151-157

ISBN : 2-84520-553-8

ISSN : $1254-4590$

Référence électronique

Antoine Prost et Odile Luginbühl, «La recherche en éducation », Revue internationale d'éducation de Sèvres [En ligne], 29 | avril 2002, mis en ligne le 25 novembre 2011, consulté le 05 juillet 2021. URL http://journals.openedition.org/ries/1939 ; DOI : https://doi.org/10.4000/ries.1939

Ce document a été généré automatiquement le 5 juillet 2021.

(c) Tous droits réservés 


\title{
La recherche en éducation
}

\author{
Entretien avec Antoine Prost
}

Antoine Prost et Odile Luginbühl

\section{NOTE DE L'ÉDITEUR}

Propos recueillis par Odile Luginbühl

Revue internationale d'éducation Sèvres: Dans quelle perspective avez-vous conduit, à la demande du ministre de l'Éducation nationale, votre réflexion sur la recherche en éducation en France?

Antoine Prost : Tout rapport ${ }^{1}$ pour un ministre a un cahier des charges. C'est une commande. On doit répondre aux questions posées. Vous avez toujours un état des lieux, indispensable, et des propositions à faire qui sont attendues. Dans le cas d'espèce, une demande supplémentaire concernait les IUFM et l'INRP.

RIES : Dans votre rapport, vous dressez un état des lieux « sans complaisance », selon les termes de M. Lang. Quelle analyse faites-vous de la situation actuelle de la recherche en éducation?

A.P. : Depuis la publication du rapport, je suis allé à Lille au congrès de l'association des enseignants-chercheurs en sciences de l'éducation. Quatre cents recherches y ont été présentées. Ce qui confirme notre diagnostic: nous ne manquons pas de recherches, il y en a beaucoup, mais elles ne servent à rien, elles ne sont pas utilisées et même ne peuvent pas l'être parce qu'elles ne sont pas coordonnées. Chacun fait la recherche qu'il a envie de faire avec les moyens dont il dispose : ici une recherche sur la lecture au cours préparatoire parce que vous avez quatre classes préparatoires sur lesquelles vous pouvez travailler... et à cent kilomètres de là, quelqu'un d'autre mène une recherche sur le traitement des élèves qui ne savent pas lire au CE1 avec cinq CE1. Que peut-on faire de ces deux recherches ? Rien. Elles ne sont pas cumulatives, car elles n'ont pas été conçues pour l'être ; elles ne sont pas menées selon les mêmes problématiques ni avec les mêmes protocoles expérimentaux. Elles sont donc 
inutilisables et vous avez des dizaines de recherches sur l'apprentissage de la lecture dont on ne peut rien faire.

Les recherches sont aussi très mal évaluées. On est ici victime de l'absence de structure de type CNRS dans le domaine des sciences de l'éducation. L'évaluation est donc faite par le ministère pour les équipes universitaires lors des contractualisations. Elle est faite par les comités scientifiques des établissements, des universités, de l'INRP, mais ces comités scientifiques n'ont pas le tranchant nécessaire et leur religion est un peu faite à l'avance.

L'évaluation est aussi fonction de la coordination qui n'est pas assurée. Il n'existe pas, au ministère de l'Éducation nationale, de structure de pilotage, pas de direction ou de mission de la Recherche, à la différence d'autres ministères. Pour l'Éducation nationale, il y a l'INRP - mais c'est un grand établissement qui n'est pas exactement dans la main du ministère -, et la direction de la programmation et du développement (DPD), qui n'a aucune mission de coordination de la recherche. Et elle ne l'assure donc pas. Alors on imagine des structures incitatives et on lance des appels d'offres. La direction de la Recherche et des Études doctorales, quand elle existait, avait lancé un très bel appel d'offres sur l'éducation, avec un très beau comité scientifique... On a autorisé une quinzaine de projets pour environ deux millions de francs. Les chercheurs avaient dix-huit mois à deux ans pour faire leurs recherches. Entre temps la DRED avait disparu. Donc les chercheurs n'avaient plus d'interlocuteurs et il n'y avait plus de comité scientifique pour se saisir des recherches et les évaluer. On a créé le Comité national de coordination de la recherche en éducation, le CNCRE. Il a fait des appels d'offres lui aussi et il a été supprimé avant qu'ils ne soient rendus. Sans compter qu'au ministère de l'Éducation nationale, on ne suit pas vraiment les appels d'offres. Je suis allé récemment au séminaire de lancement d'un des récents appels d'offres du Plan Urbanisme, Construction, Architecture, au ministère de l'Equipement. Une quinzaine de recherches avaient été retenues. Toutes les équipes concernées étaient là, pour présenter leurs recherches aux autres et ce ne sont pas les seconds couteaux qui étaient venus... C'est là qu'on voit si une structure marche. Avant la fin de ce programme, d'autres séminaires seront organisés périodiquement. Il y a là tout un suivi, tout un accompagnement des recherches, et une évaluation s'effectue à travers cette coordination. Je n'ai jamais rien vu de tel au ministère de l'Éducation nationale. Il n'a pas ce savoir faire du pilotage de la recherche, et l'on peut même dire qu'il s'en moque. En tout cas, il n'a rien fait jusqu'ici pour l'acquérir !

RIES: Quelles sont, selon vous, les questions majeures, actuellement sans réponse, qui devraient orienter prioritairement les axes de la recherche en éducation?

A.P.: Le rapport en identifie quatre. Il y en aurait peut-être cinq, ou seulement trois... Le choix de ces quatre axes ne répond pas à une procédure objective longue. Nous avons écouté les gens, parlé entre nous et voilà.

Un axe tourne autour de l'apprentissage en école élémentaire. Il me paraît désolant de se résigner à ce que $10 \%$ des élèves commencent une scolarité au collège dans des conditions qui programment leur échec, avec tout ce que cet échec signifie. Cela me paraît plus grave, à certains égards, que la "vache folle ». Pourtant, la "vache folle » a motivé un véritable effort de recherche. $10 \%$ d'enfants incapables de suivre au collège, cela n'a pas l'air de turlupiner beaucoup de gens. On dit bien : le collège doit faire quelque chose. Mais quand le problème se pose, il est déjà trop tard. Il fallait 
s'en occuper avant. Cela ne signifie pas que l'école française soit en situation d'échec dramatique. Ne dramatisons pas. Elle marche plutôt bien. Mais malgré tout, ce taux d'échec n'est pas acceptable. Si l'on entreprend d'y remédier, il faut poser toute une série de questions sur l'incidence des pratiques de classe sur les apprentissages, car les différences entre les pratiques constatées ont une telle ampleur qu'elles ne peuvent pas ne pas avoir de conséquences, bonnes ou mauvaises. Il faut s'interroger sur le lien entre ce que l'on sait en psychologie de l'apprentissage et les stimulations scolaires, c'est-à-dire évaluer les pratiques scolaires à la fois par référence à ce qu'on peut constater qu'elles produisent dans les apprentissages des enfants et les mettre en rapport avec ce qu'on sait en termes de psychologie cognitive.

Un deuxième axe tourne autour de la violence qui est l'un des grands sujets de société. La violence a été examinée exclusivement sous son angle socio-économique et les chercheurs ont trouvé qu'elle était liée aux conditions sociales, économiques et familiales que l'on imaginait. Ce constat ne débouche pas sur des remédiations, car ce n'est pas l'école qui peut lutter contre le chômage. D'autres approches, de type anthropologique ou sociologique, seraient peut-être plus fécondes. Il faut travailler spécifiquement sur les modes de gestion des établissements, leur rapport avec la violence et inversement sur ce que la violence provoque quant au mode de gestion des établissements. Egalement sur le rôle des pratiques scolaires: comment la violence les fait-elle évoluer ? Là, même d'un simple point de vue descriptif, non pas analytique, on ne sait pas exactement ce que les professeurs ont changé pour s'adapter au climat des classes particulièrement difficiles, ce qu'ils ont abandonné, ce qu'ils ont imaginé de nouveau... et en sens inverse, car tout cela est réciproque, quel est le rapport entre les pratiques pédagogiques des professeurs et la violence de leurs élèves? On peut penser que certains professeurs versent de l'huile sur le feu sans s'en rendre compte et aussi que d'autres ont un sens particulier de ce qu'il faut faire pour que le lait ne déborde pas, si vous me permettez ces métaphores culinaires. Évidemment, on n'arrivera pas à des conclusions aussi catégoriques que dans le premier cas mais je crois possible d'améliorer un peu les choses.

Le troisième axe suscitera à mon avis des difficultés avec le ministère. C'est le propre des recherches liées à des organismes administratifs. Si j'écris l'histoire de ma famille, je risque d'avoir des difficultés avec mes sœurs parce que je vais raconter des histoires qu'elles n'ont pas envie que je raconte. Peut-être vais-je devoir utiliser un pseudonyme... C'est la même chose pour l'Éducation nationale. Le ministère ne sait pas exactement ce qu'il a fait. Il a décentralisé et instauré la discrimination positive. Il ignore quel est l'écart entre les établissements les mieux dotés et les établissements les moins bien dotés. Jusqu'où va-t-il ? De 1 à 1,5 ? 1,8 ? 2,5 ? Ce n'est pas pareil ! Il ne sait pas comment les mesures de décentralisation qu'il a édictées sont exploitées à la base. A mon avis, très peu, mais ce n'est que mon opinion, non un résultat valide. Il est donc indispensable d'entreprendre une évaluation des politiques de discrimination positive et de décentralisation, ce qui ne consiste pas seulement à les décrire, à voir si elles sont appliquées ou non et quelle est leur ampleur, mais aussi à évaluer leurs effets. La politique de décentralisation devait rapprocher les établissements de leur environnement social. Est-ce que cela a été le cas? La politique de discrimination positive devait lutter contre l'échec scolaire. Est-ce que les élèves réussissent mieux quand l'établissement a plus de moyens? Les élèves des collèges surdotés obtiennent-ils de meilleurs résultats que les autres? Mais supposez 
que vous soyez ministre et que le ministère s'apprête à publier une recherche selon laquelle l'écart entre les collèges irait de un à deux. Est-ce que vous la laisseriez sortir? Non. Parce que vous avez une grève dans les quinze jours qui suivent. Il faut avoir conscience de cette difficulté inhérente à la diffusion des résultats de la recherche. Aucun ministère n'a envie d'être placé dans une situation où la recherche pourrait montrer qu'il a fait fausse route. Pourtant il est indispensable de tirer ces questions au clair : on a rompu avec une tradition séculaire et à mon avis on est allé assez loin, dans les textes du moins, mais jusqu'où, et avec quels résultats?

Quatrième axe: la recherche en formation des enseignants, la construction des professionnalités enseignantes. C'est aussi destiné à ancrer les IUFM dans la recherche, à leur permettre de nourrir la formation qu'ils organisent à partir de recherches sur la façon dont le métier d'enseignant évolue. On ne peut pas bien former à un métier si on ne se rend pas compte du champ de forces dans lequel ce métier est en train d'évoluer. Donc qu'est-ce qu'on sait sur l'évolution des pratiques enseignantes et en quoi ce que l'on pourrait savoir sur ces pratiques pourrait alimenter une formation.

Ces quatre axes ont chacun une légitimité. Le rapport ne les présente pas comme des programmes de recherche. Il dit très clairement qu'il y a toute une élaboration de ces thèmes à mener pour en tirer par exemple des appels d'offres; il faut sensibiliser des équipes, discuter avec elles, avec les différents partenaires autour de chacun de ces axes pour les préciser et en tirer quelque chose. C'est pourquoi il faut compter un an ou deux de mise en route de ce programme stratégique et l'on n'aura pas de réponse avant quatre ou cinq ans. Il faut se donner un horizon d'une quinzaine d'années parce que ce sont des questions lourdes. Au bout de deux ans, on n'aurait même pas une indication de tendance. Il faut lancer plusieurs équipes de recherche de façon coordonnée, il faut les suivre, les accompagner, faire un colloque quand elles auront rendu leurs résultats, publier ceux-ci... À ce moment-là, on commencera à voir les résultats se dégager. Ces sujets sont de grands sujets. Ils ne peuvent pas être traités sans un programme incitatif stratégique, en se donnant le temps et la stabilité nécessaires.

RIES : Au cœur de vos propositions se situe la mise en place d'un programme stratégique de recherches en éducation. Comment l'envisagez-vous?

A.P. : Nous avons dit un programme stratégique parce qu'il ne s'agit pas de lancer un appel d'offres. Il ne s'agit pas de lancer un caillou dans la mare, mais de construire une jetée. C'est un effort de longue haleine.

Il exige d'abord une instance de pilotage, une petite équipe à temps plein qui contacte les gens, organise des rencontres, des colloques, définit des appels d'offres, en rédige le texte, le fait circuler et le soumet. Les appels d'offres sont comme les maisons, comme l'architecture: jamais un architecte n'a donné une bonne réponse à une question qui ne lui avait pas été posée! Les architectes construisent selon un programme. De même, si l'appel d'offres est mal fait, les réponses seront insatisfaisantes. Ou même, il n'y aura pas de réponse du tout. Ensuite, il faut administrer les appels d'offres, veiller à leur suivi et aller voir les équipes. Le métier de directeur ou de président de commission au CNRS, c'est cela : on va voir sur place comment les équipes sont installées, ce qu'il y a dans la bibliothèque, si des étudiants passent... C'est important pour savoir si une équipe est bonne et si cela vaut la peine 
de la financer. C'est un vrai travail. Il ne suffit pas d'un président qui vient de temps en temps.

En second lieu, il faut une vraie structure scientifique parce que dans notre esprit le comité scientifique de ce programme doit pouvoir financer des recherches sur appels d'offres mais aussi sur projets. Si une équipe estime qu'elle a un projet intéressant qui mérite d'être subventionné, il faut qu'elle le soumette, qu'il soit examiné et s'il est bon, on signe un contrat. Cela suppose un fonctionnement un peu analogue à celui d'une commission du CNRS, c'est-à-dire un conseil scientifique qui se réunit plusieurs fois par an pour plusieurs jours. Un vrai travail. Des deux côtés. Les équipes qui candidatent au CNRS discutent longuement leur projet de recherche, pour le mettre au point, rendre compte des acquis, compléter les bibliographies... Les rapporteurs regardent ces comptes-rendus et ces projets, ils prennent leurs renseignements, lisent les productions de l'équipe. Quand la commission se réunit, les deux rapporteurs font leur rapport et on les discute pendant parfois plus d'une heure: d'où la qualité et la fiabilité de l'évaluation. Or si vous discutez une heure par équipe, vous ne pouvez pas examiner plus de six ou sept équipes par jour. Donc il faut un comité scientifique fort et assuré d'une certaine stabilité. Là aussi, il faudra une quinzaine d'années pour que les effets de cette politique sur la structuration de la recherche donnent tous leurs fruits.

RIES : Vous soulignez la nécessité de développer des recherches « contextualisées ». Quels sont les éléments à prendre en compte dans une telle démarche et quelles difficultés soulève-t-elle?

A.P. : Nous nous sommes efforcés d'éviter les caricatures. Par exemple, en matière de recherche en éducation, la distinction recherche fondamentale appliquée n'est guère pertinente. Une recherche portant par exemple sur la validation d'un didacticiel sur l'accord du participe passé, est en principe de la recherche appliquée. Une recherche sur la construction du groupe verbal chez l'enfant, c'est une recherche de linguistique fondamentale. Dans un cas comme dans l'autre, ou bien vous restez dans votre laboratoire ou dans votre centre de recherches, ou bien vous allez voir dans les classes, sur le terrain. Dès lors, vous constatez deux choses : le terrain est habité et il y a des acteurs sur place qui ont une volonté, une capacité d'initiative et ils ne vont pas se contenter d'appliquer les consignes des chercheurs de façon passive. Ils vont les interpréter librement. Même dans les recherches classiques, les acteurs ont toujours une part d'initiatives et il y a des élèves qui vont utiliser les consignes, les subvertir, imaginer d'autres consignes... cela se déroule dans un contexte. De plus, ces recherches portent toujours sur un processus, c'est-à-dire sur quelque chose qui se déroule dans le temps, de sorte que ce qui se passe à $\mathrm{T}+2$ est tributaire de $\mathrm{T}+1$ luimême tributaire de ce qui s'est passé au moment $T$. Donc les décisions qui ont été prises entre $\mathrm{T}$ et $\mathrm{T}+1$ et entre $\mathrm{T}+1$ et $\mathrm{T}+2$ ont une incidence sur le résultat final. Le fait que ces recherches soient nécessairement amenées à prendre en compte des contextes à la fois de temps et d'espace fait qu'elles sont plus compliquées. Il y a plus de contraintes. L'administration de la preuve est plus difficile parce que les paramètres sont plus nombreux. Il faut donc plus d'ingéniosité. C'est pourquoi on a proposé de les appeler «recherches contextualisées », étant évident qu'il n'y a pas qu'en sciences de l'éducation que certaines recherches sont contextualisées. Les recherches médicales le sont également, la recherche architecturale, la recherche sociologique quand elle n'est pas purement quantitative...et même l'histoire la plus contemporaine quand elle est confrontée aux personnes dont elle raconte les actes. 
Ce n'est pas propre aux recherches en éducation mais cette contextualisation y est plus générale et plus contraignante. L'évaluation est rendue plus difficile et doit donc être plus rigoureuse.

RIES: Dans la perspective d'une meilleure articulation entre la recherche et le système éducatif, vous développez le concept de "culture-recherche ». Comment le définissez-vous et quelles en sont les modalités concrètes ?

A.P.: Si les chercheurs et les praticiens ou les décideurs, comme on dit, se rencontrent seulement quand les uns ont un problème à poser aux autres, cela ne peut pas marcher. Les temporalités et les problématiques ne sont pas les mêmes. Ces deux mondes ne sont pas exactement dans la continuité l'un de l'autre. Pour qu'ils se rencontrent efficacement, il faut organiser leur rencontre sur des terrains communs. C'est ce que nous avons souligné en relevant la nécessité de développer ce que nous avons appelé une " culture-recherche ", c'est-à-dire une sensibilisation des praticiens et des décideurs à la recherche et inversement des chercheurs aux problèmes des praticiens et des décideurs. Ce qui nous a amenés à souligner l'importance de « lieux intermédiaires » où puisse s'épanouir cette culture-recherche. Il ne faut pas compter seulement sur les bénévoles pour animer ces lieux intermédiaires : il y en a toute une série, et ils doivent tous être cultivés, soutenus, entretenus... Ce peuvent être des lieux de formation, des écoles d'été, des mouvements pédagogiques militants, ce peut être aussi ce que certains recteurs développent autour d' «Innovalo " ${ }^{2}$. Les IUFM peuvent jouer un rôle non seulement avec la formation permanente mais en organisant des cycles de conférence, des débats. Il faut faire vivre un milieu d'échanges sur les problèmes d'enseignement avec une approche de recherche. C'est de ce tissu que peut naître une fécondation réciproque de la recherche par la pratique et de la pratique par la recherche.

RIES: À plusieurs reprises vous dénoncez ce que vous appelez «l'hexagonalisme» de la recherche française en éducation. Quel rôle attribuez-vous à l'ouverture internationale en matière de recherche et selon quelles modalités peut-elle s'effectuer?

A.P. : Il faut remettre la France dans le courant des recherches anglo-saxonnes. Et aussi dans le courant des recherches canadiennes, belges et suisses. Mais là, les échanges sont meilleurs : il n'y a pas l'obstacle de la langue. Avec quels moyens? C'est simple. Il faut envoyer des doctorants et des post-doctorants dans les laboratoires étrangers. C'est ce que je fais avec mes doctorants en histoire. Quand je peux les envoyer à Harvard ou à Cambridge, je le fais. Et ils n'y passent pas toujours des semaines paradisiaques! Je trouve qu'il faudrait organiser des colloques internationaux comme le CNRS l'a fait au lendemain de la guerre où la recherche avait par force été coupée de tous les développements extérieurs. On a organisé des colloques internationaux pour faire connaître aux chercheurs français les résultats des Américains notamment. Si des chercheurs vont à l'étranger, il se passera ce qui se passe dans tous les secteurs, c'est-à-dire des jeunes qui parlent anglais ou allemand, qui seront en relation par Internet avec les Américains, les Anglais, les Néerlandais etc. ... Il y aura des rencontres, des newsletters se mettront à circuler, mais il faut de l'argent pour organiser des colloques internationaux et pour envoyer des gens à l'étranger. Cela passe probablement par des jumelages. Un exemple : on commence à avoir de bons historiens des États-Unis en France, alors que ce n'était pas le cas dans ma génération ou alors c'était rarissime. La rue d'Ulm a conclu un accord avec Harvard et, tous les ans, un normalien passe une année au centre d'Études européennes de Harvard. Au bout de vingt ans, les résultats se voient... C'est comme 
quand vous plantez des arbres. Ils ne prennent pas tous. Il faut en replanter beaucoup. Certains repoussent. Certains sont bons et poussent plus vite...

RIES: Quelles sont d'ores et déjà les mesures prises par le ministère de l'Éducation nationale à la suite de votre rapport ?

A.P. : Marie Duru-Bellat et Denis Meuret ont été nommés pour piloter ce programme. C'est un très bon choix qui n'est pas politique et qui n'est pas de court terme. Le ministre ne tirera pas personnellement bénéfice des mesures qu'il a prises. Il faut lui rendre cette justice. Je constate qu'il a dégagé déjà dix millions de francs au budget 2002. Cela permet de commencer. Quand vous comparez ces dix millions aux trois millions de la DPD, c'est un effort significatif. La décision de publier le rapport est également bien venue : si l'on veut que ce programme interdisciplinaire de recherche ait un avenir quel que soit le résultat des élections, il faut lui donner une légitimité. Il ne peut la trouver que si l'argumentation - rigoureuse je le crois - qui conduit à cette conclusion est sur la place publique et se défend d'elle-même.

\section{NOTES}

1. Le rapport «Pour un programme stratégique de recherche en éducation » a été remis aux ministres de l'Éducation nationale et de la Recherche en juillet 2001. Il est consultable sur le site Internet du ministère : www.education.gouv.fr

2. Innovalo : mission ou groupe de travail spécifique existant dans la plupart des académies en France, destiné à promouvoir et diffuser les expériences pédagogiques innovantes.

\section{AUTEURS}

\section{ANTOINE PROST}

Antoine Prost est un historien de la société française au XX ${ }^{\mathrm{e}}$ siècle à travers notamment l'étude des groupes sociaux, des institutions et des mentalités. Spécialiste des questions d'éducation, il a collaboré à plusieurs reprises à la définition des politiques d'éducation depuis 1964.

\section{ODILE LUGINBÜHL}

Odile Luginbühl, IA-IPR de lettres, est rédactrice en chef de la Revue internationale d'éducation de Sèvres 